\title{
Usage de la refusion par bombardement électronique pour la purification et le recyclage des alliages
}

\section{Application of the electron beam remelting for the purification and the recycling of alloys}

\author{
Jean-Pierre Bellot, Jonathan Martens, Julien Jourdan et Alain Jardy \\ Institut Jean Lamour, UMR 7198, CNRS ('LabEx DAMAS')/ Université de Lorraine, 54011 Nancy, France
}

\begin{abstract}
Among the ultimate purification techniques applied to the high melting point metallic materials, the electron beam remelting process, combining a high thermal power and vacuum, is particularly conducive to volatilization. This paper describes the technique and its efficiency to remove impurities by distillation.
\end{abstract}

Résumé. Parmi les techniques de purification ultime de métaux à hauts points de fusion, le procédé de refusion par bombardement électronique associe une puissance thermique garantissant la fusion du métal, et une refusion sous vide permettant l'élimination d'impuretés par distillation. Ce papier présente la technique et le potentiel d'élimination par distillation.

\section{INTRODUCTION}

Parmi les alliages réactifs ou réfractaires, une majorité d'entre eux présente un intérêt évident au recyclage ; pour des raisons économiques car l'élaboration de ces alliages est complexe et énergétiquement onéreuse (le titane est un parfait exemple), ou pour des raisons stratégiques du fait de la raréfaction ou de l'accessibilité des ressources minières [1] (Niobium, Tantale, Tungstène par exemple). L'usage de ces matériaux requiert le plus souvent une haute pureté, et donc la filière de recyclage nécessite une étape de purification ultime. Le procédé de refusion par bombardement électronique (BE) apporte la puissance enthalpique pour fondre ces métaux à hauts points de fusion, protège le métal de toute pollution grâce à l'emploi de creusets en cuivre refroidis par eau et enfin l'application d'un vide secondaire permet d'éliminer certaines impuretés par distillation. C'est ce dernier point qui est examiné dans ce papier.

\section{LE PROCÉDÉ}

La technologie de la fusion par bombardement électronique a été industriellement pratiquée depuis le début des années 50. Les premières applications concernaient la purification du niobium et du tantale. Plus récemment, cette technologie est devenue plus attractive du fait de sa capacité à recycler et à purifier le titane et ses alliages, en particulier pour des applications aéronautiques. Des développements voient actuellement le jour pour étendre ce domaine d'application aux superalliages (IN 718, René $95, \ldots)$.

Deux procédés d'élaboration utilisant comme moyen de fusion un (ou des) canons(s) à électrons se sont développés à ce jour :

- le procédé discontinu « Drip Melting» pour lequel un lingot primaire est fondu par le faisceau $\mathrm{BE}$

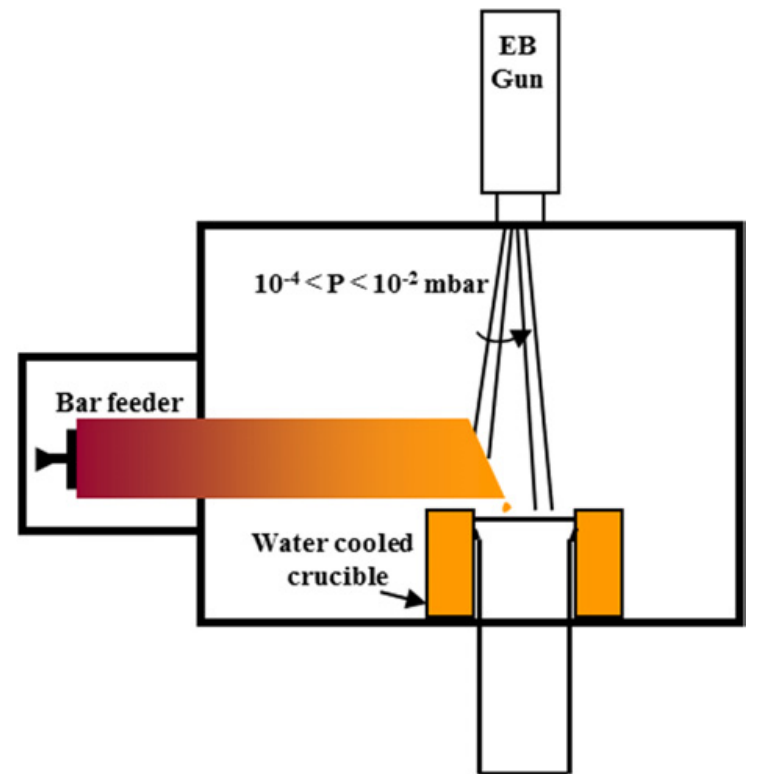

Figure 1. Schéma du procédé Drip Melting.

sous forme de gouttes qui viennent former un lingot secondaire (Fig. 1),

- le procédé continu «Electron Beam Cold Hearth Refining »(EBCHR) basé sur la séparation des étapes de purification et de solidification, et pour lequel un creuset à fond plat (Cold Hearth) est utilisé notamment pour éliminer les inclusions par décantation et/ou dissolution.

L'ensemble de ces opérations est réalisé dans une enceinte sous vide secondaire, ce qui permet un bon dégazage du métal.

Ces procédés de refusion par BE présentent les avantages suivants :

- absence de réfractaire (ce qui évite la pollution du métal liquide), 
- bonne élimination des éléments volatils (présence du vide secondaire), des inclusions de faible et haute densité dans le four à surverse (procédé EBCHR),

- bon contrôle du flux énergétique en termes de puissance et de localisation, ce qui permet un meilleur contrôle de la solidification et du hottopping,

- souplesse quant à l'alimentation en matière première (recyclage de déchets).

\section{PURIFICATION PAR DISTILLATION SOUS VIDE}

\section{Equilibre thermodynamique}

$\mathrm{Si}$ on considère un soluté $\mathrm{j}$ en solution dans un bain de matrice $\mathrm{M}$ liquide, la densité de flux net molaire d'évaporation s'exprime [2,3] selon la loi de Langmuir :

$$
\varphi_{\mathrm{j}}=\frac{1}{\sqrt{2 \pi \mathbf{R} \mathbf{T} \mathbf{M}_{\mathrm{j}}}} \mathbf{P}_{\mathbf{j}}^{\mathbf{e q}}
$$

A l'interface, l'équilibre thermodynamique local s'exprime en appliquant la loi de Henry :

$$
\mathbf{P}_{\mathbf{j}}^{\mathbf{e q}}=\gamma_{\mathbf{j}} \mathbf{x}_{\mathbf{j}} \mathbf{P}_{\mathbf{j}}^{\circ}
$$

$\gamma_{i}$ est ici le coefficient d'activité. En ce qui concerne la matrice $\mathrm{M}$, la pression de vapeur d'équilibre au-dessus de la surface du liquide est donnée par :

$$
\mathbf{P}_{\mathbf{M}}^{\mathrm{eq}}=\mathbf{a}_{\mathbf{M}} \mathbf{P}_{\mathbf{M}}^{0}=\left(1-\sum_{\mathbf{j}} \mathbf{x}_{\mathbf{j}}\right) \mathbf{P}_{\mathbf{M}}^{0} ; \mathbf{P}_{\mathbf{M}}^{0}
$$

Le rapport des densités de flux molaires de Langmuir entre le soluté $\mathrm{j}$ et la matrice $\mathrm{M}$ donne :

$$
\frac{\varphi_{\mathbf{j}}}{\varphi_{\mathbf{M}}}=\mathbf{x}_{\mathrm{j}} \gamma_{\mathbf{j}} \frac{\mathbf{P}_{\mathbf{j}}^{0}}{\mathbf{P}_{\mathbf{M}}^{0}} \sqrt{\frac{\mathbf{M}_{\mathbf{M}}}{\mathbf{M}_{\mathbf{j}}}}=\mathbf{x}_{\mathbf{j}} \cdot \mathbf{s}(\mathbf{j}, \mathbf{M})
$$

avec s, coefficient de partage (ou de séparation) entre la phase raréfiée et le liquide, qui a la particularité de ne dépendre que de la température et des propriétés thermochimiques du mélange $\mathrm{j}$ dans $\mathrm{M}$. La purification de l'impureté j selon le procédé par BE ne pourra être obtenue par distillation que si $\mathrm{s}>1$.

On peut aussi étudier comment varie le facteur de séparation s avec la température de surface du bain, à partir de la formule de Clapeyron. On obtient alors :

$$
\frac{\mathbf{d} \ln \mathbf{s}}{\mathbf{d T}}=\frac{\mathbf{d} \ln \left(\frac{\mathbf{P}_{\mathrm{j}}^{0}}{\mathbf{P}_{\mathrm{M}}^{0}}\right)}{\mathbf{d T}}=\frac{\Delta \mathbf{H}_{\mathrm{vj}}-\Delta \mathbf{H}_{\mathrm{vM}}}{\mathbf{R T}^{2}}
$$

L'effet de la température dépendra donc du signe du numérateur. Le tableau 1 compare les enthalpies massiques d'évaporation au point d'ébullition de chaque soluté.

Si on s'intéresse à la purification du niobium pollué par les trois éléments présentés dans le tableau 1, on observe qu'une augmentation de la température n'est pas favorable à la distillation.
Tableau 1. Enthalpies d'évaporation.

\begin{tabular}{|c|c|c|c|c|}
\hline & Fe & Ti & Mo & Nb \\
\hline$\Delta \mathrm{H}_{v}(\mathrm{~kJ} / \mathrm{mol})$ & 345 & 411 & 578 & 659 \\
\hline
\end{tabular}

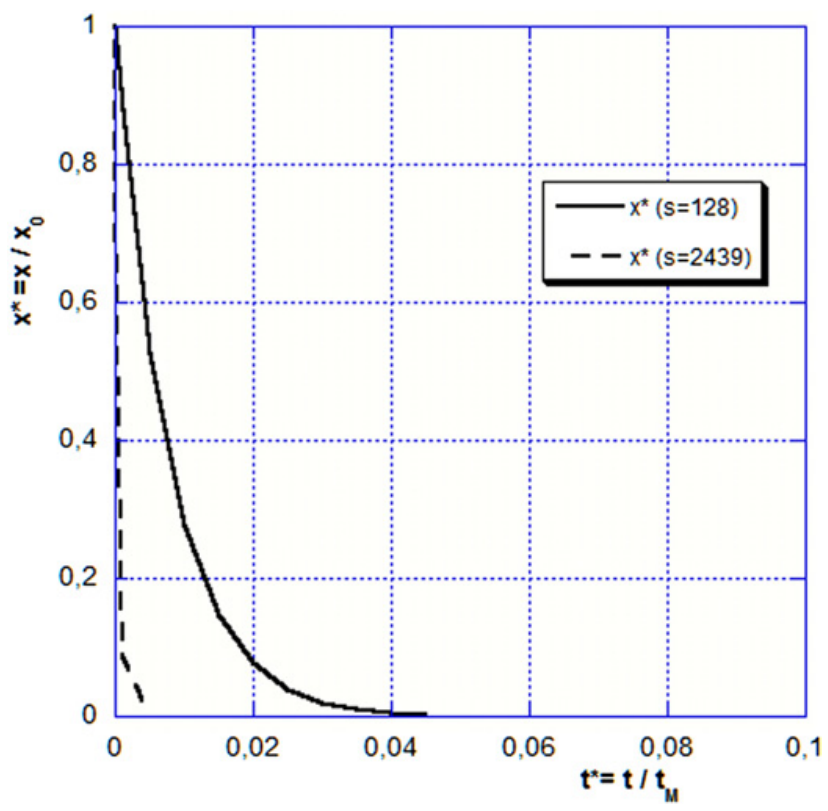

Figure 2. Cinétique d'élimination de titane (trait continu) et fer (pointillé) dans du niobium.

\section{CINÉTIQUE DE DISTILLATION}

On considère un bain de surface $\mathrm{S}$ où s'effectue l'évaporation des espèces $\mathrm{j}$ et $\mathrm{M}$. Les bilans molaires du soluté et de la matière totale dans le bain s'expriment respectivement :

$$
\frac{\mathbf{d n}_{\mathrm{j}}}{\mathbf{d t}}=-\mathbf{S} \varphi_{\mathrm{j}} \text { et } \frac{\mathbf{d n} \mathbf{n}_{\mathrm{t}}}{\mathbf{d t}}=-\mathbf{S}\left(\varphi_{\mathrm{M}}+\varphi_{\mathrm{j}}\right)
$$

En introduisant le temps $t_{M}$ total pour évaporer tout le bain, et en appliquant la relation (4), les bilans (6) se résolvent facilement pour obtenir la relation :

$$
\mathbf{x}_{\mathbf{j}}(\mathbf{t})=\mathbf{x}_{\mathbf{j} 0}\left(1-\frac{\mathbf{t}}{\mathbf{t}_{\mathbf{M}}}\right)^{\mathrm{s}-1}
$$

La relation (7) démontre que l'élimination du soluté j dans la matrice $\mathrm{M}$ n'est possible que si $\mathrm{s}>1$, et que sa vitesse croit à la puissance $(\mathrm{s}-1)$.

Donnons un exemple d'application avec la purification par BE de niobium pollué par des traces de titane et de fer. La figure 2 donne l'évolution de $\mathrm{x}^{*}=\mathrm{x}_{\mathrm{j}} / \mathrm{x}_{\mathrm{j} 0}$ en fonction du temps $\mathrm{t}^{*}=\mathrm{t} / \mathrm{t}_{\mathrm{M}}$. Pour ces deux exemples, les coefficients d'activités ont été pris égaux à 0,1 . On note que l'élimination du fer est immédiate et totale, tandis que pour le titane, le titre aura atteint moins de $10 \%$ de sa valeur initiale au bout d'un temps $t=0,02 t_{M}$.

L'Institut Jean Lamour dispose d'un four de laboratoire de $100 \mathrm{~kW}$, dans lequel des essais de purification de métaux pollués sont mis en œuvre. Les résultats expérimentaux confortent l'approche théorique présentée ici. 


\section{CONCLUSION}

Dans le procédé de refusion par BE, la combinaison des hautes températures atteintes en surface et d'un vide secondaire favorise l'élimination des éléments à fortes pressions de vapeur. Cette élimination est rendue possible si le coefficient de séparation $\mathrm{s}$ (Eq. (4)) est supérieur à l'unité, et la cinétique d'élimination s'exprime selon une loi à la puissance (s-1).

\section{Références}

[1] Working Group, Critical raw materials for the EU, July, (2010), http://ec.europa.eu/enterprise/policies/ rawmaterials, documents/index_en.htm

[2] L. Langmuir, Phys. Rev. 2, (1913), 329

[3] J.P. Bellot, H. Duval, M. Ritchie , A. Mitchell, D. Ablitzer, Evaporation of $\mathrm{Fe}$ and $\mathrm{Cr}$ from Inductionstirred austenitic stainless steel: Influence of the inert gas pressure, ISIJ, Vol. 41 (2001), №7, pp. 696-705. 